\title{
VECSEL platform for single/dual frequency CW operation and ultrashort pulse generation
}

\author{
A. Laurain ${ }^{1}$, R. Rockmore ${ }^{1}$, I. Kilen ${ }^{1}$, C. Baker ${ }^{1}$, M. Scheller ${ }^{1}$, S. Addamane ${ }^{2}$, G. Balakrishnan ${ }^{2}$, R. J. Jones ${ }^{1}$ \\ S.W. Koch ${ }^{1,3}$, and J. V. Moloney ${ }^{1}$, \\ ${ }^{1}$ College of Optical Sciences, University of Arizona, 1630 E. University Blvd, Tucson AZ 85721, ${ }^{2}$ Center for High Technology Materials, \\ University of New Mexico, Albuquerque, NM 87106, ${ }^{3}$ Physics and Material Sciences center, Philipps Universitat, Marburg, Germany. \\ Email: alaurain@optics.arizona.edu
}

Abstract: We present state of the art results obtained with numerically optimized VECSEL devices for the generation of multi-Watt single frequency and multi-color operation in the near infrared as well as for the direct generation of sub-100fs modelocked pulses at multi-GHz repetition rate.

\section{INTRODUCTION}

After two decades of research and development, Vertical External Cavity Surface Emitting Lasers (VECSEL) are emerging as the technology of choice for numerous applications. They can provide multi-Watt CW outputs with extremely low intensity and frequency noise (sub-MHz linewidth), or generate ultrashort pulses in a multi-GHz repetition rate modelocked regime with peak powers exceeding multiple kilowatts. Their potential applications are further extended via non-linear intracavity frequency conversion (second and fourth harmonic generation, difference frequency generation,...) extending the wavelength range into the visible and $\mathrm{UV}$, and yielding widely tunable Terahertz and mid-IR sources via DFG. Conventional edge emitting diodes delivering high output power $(>1 \mathrm{~W})$ exhibit low brightness (incoherent) with highly divergent and asymmetrical beams. On the contrary, VECSELs exhibit circular, low divergence beams with a quality factor often approaching the diffraction limit $\left(\mathrm{M}^{2} \sim 1\right)$, ideal for imaging or coupling into fiber amplifiers. One advantage of optically-pumped VECSELs is the possibility to increase the pump and lasing mode diameter. This reduces the thermal impedance of the device and increases the laser induced damage threshold, yielding higher output power while maintaining a good beam quality [1]. Here, we demonstrate the unmatched versatility of VECSELs, showing a low noise tunable single frequency operation with more than $15 \mathrm{~W}$ of output power, a stable multi-color $\mathrm{CW}$ operation with more than $2.6 \mathrm{~W}$ of average power, and a modelocked operation with the first experimental demonstration of sub-100fs pulse duration directly from a semiconductor laser.

\section{EXPERIMENTAL METHODS}

The VECSEL gain media used in this work were grown by metal-organic vapor phase epitaxy on GaAs substrate for an emission wavelength around $1 \mu \mathrm{m}$. They consist of an InGaP confinement layer, followed by an active region based on InGaAs/GaAs(P) Quantum-Wells (QW), and a high-reflectivity AlAs/AlGaAs Bragg mirror. The number and individual placement of QWs in the active region were numerically optimized using a microscopic many-body theory [2]. For single frequency operation, 10 QWs are placed periodically on 10 antinodes of the field (resonant periodic gain, or RPG) to avoid spatial hole burning and spectral broadening, whereas for modelocked operation 12 QWs are distributed non-uniformly on 3 antinodes to broaden the gain and increase the gain saturation fluence. In each case, the structure was pumped in the $\operatorname{GaAs}(\mathrm{P})$ barriers by an $808 \mathrm{~nm}$ fiber-coupled diode module. The cavity geometries investigated were designed to provide optimal performance in each regime and are shown on Figure 1.

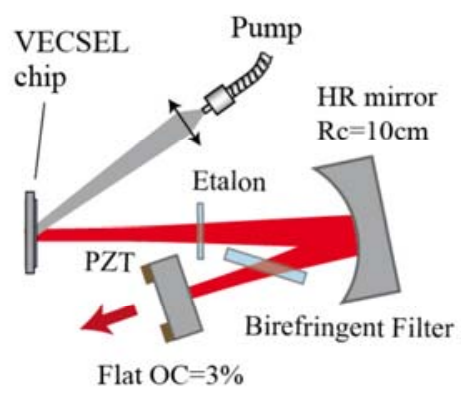

(a)

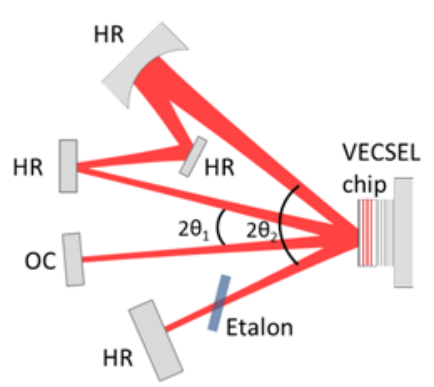

(b)

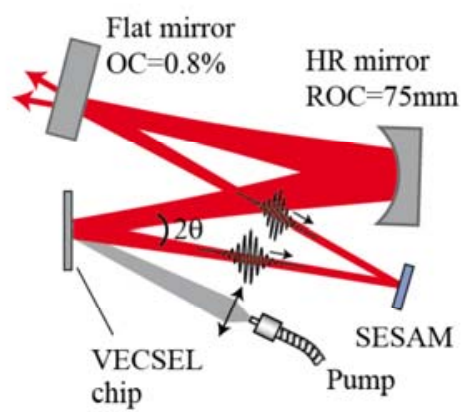

(c)

Figure 1: Cavity geometries investigated. (a) V-shaped cavity for single frequency operation. (b) Multi-fold cavity for multicolor operation. (c) Ring cavity for colliding pulse modelocked operation. 
With the cavity (a), we obtained up to $15.6 \mathrm{~W}$ in a single longitudinal and $\mathrm{TEM}_{00}$ transverse mode. The beam parameter $\mathrm{M}^{2}$ was below 1.2 for both axes confirming a near diffraction limited beam in spite of a poor quality elliptical and multimode beam from the pump diode. The free running spectral linewidth over an integration time of $1 \mathrm{~ms}$ was about $21 \mathrm{kHz}$, mostly limited by mechanical noise and pump intensity fluctuation [3]. The tuning curve of this single frequency laser is shown on Figure 2.(a). For multi-color operation with a spectral separation of a few nanometers (i.e. for $\mathrm{THz}$ spacing), we must prevent the desired modes from competing for the gain and suppress undesired ones, such as with an etalon. With the multi-fold cavity (b), the two incidence angles on the chip generates a wavelengthdependent interference pattern where the intracavity field interacts with the gain. This pattern decreases the spatial overlap of the modes corresponding to the two wavelengths and thus decreases the gain competition between them. This cavity geometry greatly improves the stability and simultaneity of the 2-color operation when compared to a Vshaped cavity [4]. The optical spectrum of the multi-fold cavity at an output power of $2.6 \mathrm{~W}$ is shown on Figure 2.(b).

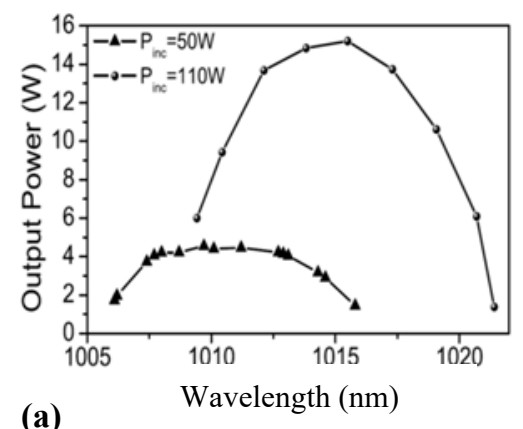

(a)

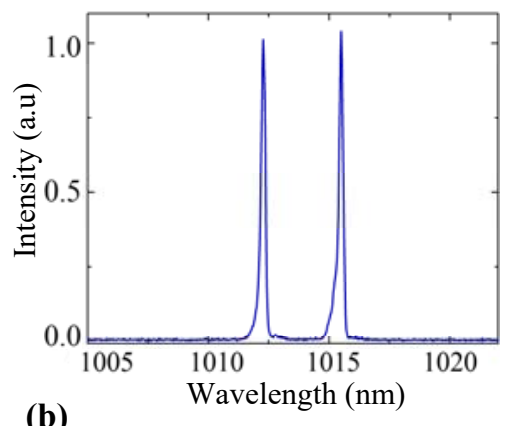

(b)

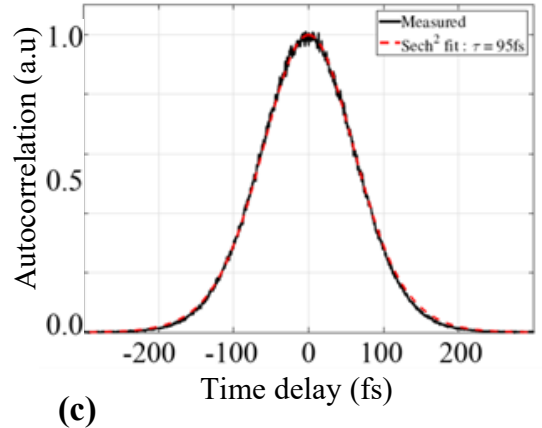

(c)

Figure 2: Experimental results from the three cavities investigated. (a) Tunability curve of a single frequency VECSEL at different pump power. (b) Bi-color optical spectrum from a multi-fold cavity VECSEL. (c) Non-collinear Second Harmonic Generation autocorrelation of a colliding pulse modelocked VECSEL fitted with a $\operatorname{sech}^{2}$ pulse with a FWHM of 95 fs.

Finally, by using a semiconductor saturable absorber mirror (SESAM) in the cavity, it is possible to passively lock the modes of the VECSEL to generate ultrashort pulses. With a carefully designed VECSEL structure based on a microscopic many-body theory, we obtained the first experimental demonstration of sub-100fs pulse duration directly from a semiconductor laser. The autocorrelation trace is shown on Figure 2.(c) The semiconductor gain and absorber structures used in the experiment were numerically optimized by modelling the pulse formation dynamics of the system. The ring shaped cavity geometry adopted was shown to improve the robustness of the modelocking regime and decrease the saturation fluence of the absorber [5]. It produced an output power of $90 \mathrm{~mW}$ per beam at a repetition rate of $2.2 \mathrm{GHz}$ with a record pulse duration of $95 \mathrm{fs}$ at a central wavelength of $1026 \mathrm{~nm}$.

These results clearly illustrate the versatility and great potential of VECSEL for photonic applications.

\section{ACKNOWLEDGMENT}

We would like to thank Wolfgang Stolz and Antje Ruiz Perez at Philipps Universität, Marburg for growing our VECSELs. This material is based upon work supported by the Air Force Office of Scientific Research under award ID FA9550-17-1-0246.

\section{References}

[1] T.-L. Wang, B. Heinen, J. Hader, C. Dineen, M. Sparenberg, A. Weber, B. Kunert, S. Koch, J. Moloney and M. \&. S. W. Koch, Laser \& Photonics Reviews, vol. 6, pp. L12-L14, 2012.

[2] J. Hader, M. Scheller, A. Laurain, I. Kilen, C. Baker and J. V. \&. K. S. W. Moloney, Semiconductor Science and Technology, vol. 32, p. 013002, 2017.

[3] A. Laurain, C. Mart, J. Hader, J. Moloney and B. \&. S. W. Kunert, Photonics Technol. Lett. IEEE, vol. 26, pp. 131-133, 2014.

[4] C. Baker, M. Scheller, A. Laurain, H.-J. Yang, A. Ruiz Perez, W. Stolz, S. J. Addamane, G. Balakrishnan and R. J. \&. M. J. V. Jones, Proc. SPIE, 2017, vol. 10087, pp. 100870B-10, 2017.

[5] A. Laurain, R. Rockmore, H.-T. Chan, J. Hader, S. W. Koch, A. R. Perez and W. \&. M. J. V. Stolz, J. Opt. Soc. Am. B, OSA, vol. 34, p. 329, 2017. 